\title{
ROBUST DIGITAL CONTROL USING POLE PLACEMENT WITH SENSITIVITY FUNCTION SHAPING METHOD
}

\author{
I.D. LANDAU* AND A. KARIMI \\ Laboratoire d'Automatique de Grenoble, ENSIEG, BP. 46, 38402 Saint Martin d'Hères, France
}

\begin{abstract}
SUMMARY
It is shown in this paper that based on identified discrete-time models a robust digital linear controller can be designed using the pole placement method combined with sensitivity function shaping in the frequency domain. Two different design techniques are presented. The first one is based on the shaping of the sensitivity functions using the fixed parts in the controller and the auxiliary poles of the closed loop while keeping the dominant poles in the desired places. The main idea of the second one is to determine a weighting filter for the output sensitivity function in an $H_{\infty}$ optimization approach which assures partial pole placement and desired performances. In this technique the weighting filter is interpreted as the inverse of the desired output sensitivity function and is computed using a constrained optimization program. The application of this technique on a flexible transmission system is presented. (C) 1998 John Wiley \& Sons, Ltd.
\end{abstract}

Key words: digital control; robust control; pole placement; $H_{\infty}$ optimization

\section{INTRODUCTION}

The controller design methodology considered in this paper is based on pole placement combined with the shaping of the sensitivity function. The computation of the controller in the pole placement technique requires the specification of the desired closed-loop poles (the nominal stability problem) and of some fixed parts of the controller for the rejection of disturbances at various frequencies (the nominal performance problem). However, this is not enough to guarantee the robustness of the design with respect to the plant model uncertainties (the robust stability and robust performance problem). A robust controller design requires also the shaping of the sensitivity functions. The sensitivity functions, particularly the output sensitivity function, are key indicators for the nominal and robust performance as well as for the robust stability of the closed-loop system. The inverse of the maximum value of the output sensitivity function, i.e., the inverse of its $H_{\infty}$ norm, gives the minimum distance between the Nyquist plot of the open-loop system and the critical point $[-1, j 0]$. This quantity, called the modulus margin, is a much more significant robustness indicator than the phase and gain margins. On the other hand, conditions for assuring a certain delay margin which is also a very important robustness indicator, particularly in the high frequency region, can also be expressed in terms of the shape of the output sensitivity function. It seems therefore reasonable to combine the pole placement with the shaping of the output

\footnotetext{
* Correspondence to: I.D. Landau, Laboratoire d'Automatique de Grenoble, ENSIEG, BP. 46, 38402 Saint Martin d'Héres, France
}

CCC 1049-8923/98/020191-20\$17.50

(C) 1998 John Wiley \& Sons, Ltd. 
sensitivity function (or eventually with other sensitivity functions) in order to design robust digital controllers for SISO plants.

This paper addresses the robust controller design problem for two cases:

(a) Only one plant model is available. In this case we consider certain robustness margins which translate into templates for the sensitivity functions.

(b) Several plant models are given or an estimation of the model uncertainty in the frequency domain is available. In this case again the uncertainties will translate into templates for the sensitivity functions.

For this purpose two different techniques are given. In the first one an iterative method is presented for correctly specifying the desired closed-loop poles and the fixed parts of the controller in order to assure the nominal performance, the desired robustness margins, as well as the robust stability of the closed loop for given classes of plant model uncertainties. This method has been applied on a large number of real systems. ${ }^{1-4}$ In the second one a systematic procedure to compute a controller based on partial pole placement with sensitivity function shaping via $H_{\infty}$ optimization is presented. In this technique the weighting filter in the $H_{\infty}$ approach is interpreted as the inverse of the desired output sensitivity function. Therefore, all of the conditions and the constraints on the output sensitivity function can be considered for the inverse of the weighting filter. One of the properties of the output sensitivity function is that it satisfies the Zames-Francis equality. ${ }^{5,6}$ This equality then is used as a performance index in a constrained optimization program in order to find the unknown parameters of the desired sensitivity function. Once the desired sensitivity function is obtained, an $H_{\infty}$ approach is used for computing the controller. The main differences between this technique and the partial pole placement presented in References 7 and 8 are as follows:

- The weighting filter is interpreted as the inverse of the desired output sensitivity function.

- This filter is the result of an optimization problem so it is not necessary to find the parameters of the filter by trial and error.

- The controller design can be carried out using only one weighting filter.

- The conditions on the weighting filter in order to obtain a stable controller are introduced.

The paper is organized as follows: Section 2 reviews the R-S-T controller and the notation. Section 3 gives the various sensitivity functions associated with the R-S-T controller. In Section 4 a template for the output sensitivity function is defined. The shaping of the sensitivity function with pole placement technique is described in Section 5. The shaping of the sensitivity function with partial pole placement using $H_{\infty}$ optimization is presented in Section 6. The application of this technique on a flexible transmission system is given in Section 7. Section 8 gives the concluding remarks.

\section{R-S-T CONTROLLER}

Figure 1 shows a schematic diagram of an R-S-T controller. The discrete time plant is described by the following transfer operator:

$$
H\left(q^{-1}\right)=\frac{q^{-d} B\left(q^{-1}\right)}{A\left(q^{-1}\right)}
$$




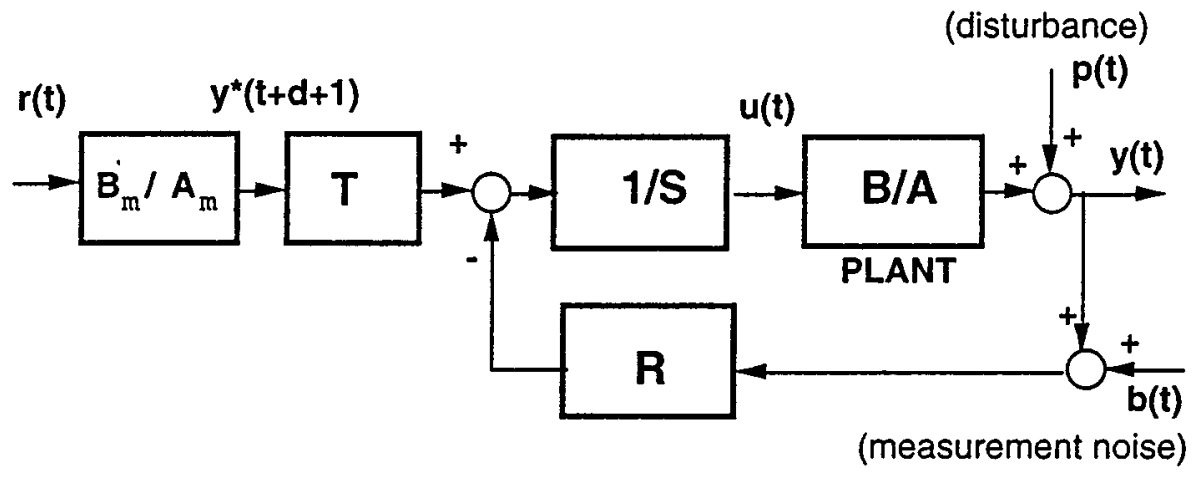

Figure 1. Closed-loop system with an $R-S-T$ controller in the presence of output disturbances and measurement noise

where $q^{-1}$ is the backward shift operator, i.e., $y(t-1)=q^{-1} y(t), d$ is the integer number of sampling periods $\left(T_{s}\right)$ contained in the plant pure time delay and

$$
\begin{aligned}
& A\left(q^{-1}\right)=1+a_{1} q^{-1}+\cdots+a_{n_{A}} q^{-n_{A}} \\
& B\left(q^{-1}\right)=b_{1} q^{-1}+b_{2} q^{-1}+\cdots+b_{n_{B}} q^{-n_{B}}
\end{aligned}
$$

It is assumed that the polynomials $A\left(q^{-1}\right)$ and $B\left(q^{-1}\right)$ do not have common factors. The canonical form of the R-S-T controller is given by

$$
S\left(q^{-1}\right) u(t)=T\left(q^{-1}\right) y^{*}(t+d+1)-R\left(q^{-1}\right) y(t)
$$

where $u(t)$ is the plant input, $y(t)$ is the plant output and $y^{*}(t+d+1)$ is the desired tracking (reference) trajectory. This trajectory may be generated by a tracking reference model.

$$
y^{*}(t+d+1)=\frac{B_{m}\left(q^{-1}\right)}{A_{m}\left(q^{-1}\right)} r(t)
$$

where $r(t)$ is the reference signal. The closed-loop transfer function between the reference trajectory and the plant output is given by

where

$$
H_{C L}\left(z^{-1}\right)=\frac{z^{-d} B\left(z^{-1}\right) T\left(z^{-1}\right)}{P\left(z^{-1}\right)}
$$

$$
P\left(z^{-1}\right)=A\left(z^{-1}\right) S\left(z^{-1}\right)+z^{-d} B\left(z^{-1}\right) R\left(z^{-1}\right)=P_{D}\left(z^{-1}\right) \cdot P_{F}\left(z^{-1}\right)
$$

defines the closed-loop poles. $P_{D}\left(z^{-1}\right)$ and $P_{F}\left(z^{-1}\right)$ correspond to the dominant and auxiliary closed-loop poles, respectively.

\section{THE SENSITIVITY FUNCTIONS}

The sensitivity functions play a crucial role in the robustness analysis of the closed-loop system with respect to modelling errors. These functions will be shaped in order to assure nominal performance for the rejection of the disturbances and the stability of the closed-loop system in the presence of model mismatch. Two types of disturbances are considered: output disturbance and measurement noise. 
The various sensitivity functions can be established from Figure 1. The transfer function between the disturbance $p(t)$ and the plant output $y(t)$ (output sensitivity function) is given by

$$
S_{y p}\left(z^{-1}\right)=\frac{A\left(z^{-1}\right) S\left(z^{-1}\right)}{A\left(z^{-1}\right) S\left(z^{-1}\right)+z^{-d} B\left(z^{-1}\right) R\left(z^{-1}\right)}=\frac{A\left(z^{-1}\right) S\left(z^{-1}\right)}{P\left(z^{-1}\right)}
$$

This function is often called simply the sensitivity function in the literature and is denoted by $S$. The transfer function between the disturbance $p(t)$ and the plant input $u(t)$ (input sensitivity function) is given by

$$
S_{u p}\left(z^{-1}\right)=\frac{-A\left(z^{-1}\right) R\left(z^{-1}\right)}{A\left(z^{-1}\right) S\left(z^{-1}\right)+z^{-d} B\left(z^{-1}\right) R\left(z^{-1}\right)}=\frac{-A\left(z^{-1}\right) R\left(z^{-1}\right)}{P\left(z^{-1}\right)}
$$

This function is often denoted by $U$ in the literature. The transfer function between the measurement noise $b(t)$ and the plant output $y(t)$ (noise sensitivity function) is given by

$$
S_{y b}\left(z^{-1}\right)=\frac{-z^{-d} B\left(z^{-1}\right) R\left(z^{-1}\right)}{A\left(z^{-1}\right) S\left(z^{-1}\right)+z^{-d} B\left(z^{-1}\right) R\left(z^{-1}\right)}=\frac{-z^{-d} B\left(z^{-1}\right) R\left(z^{-1}\right)}{P\left(z^{-1}\right)}
$$

This function with a positive sign is called the complementary sensitivity function denoted by $T$ in the literature. From equations (8) and (10) it is obvious that

$$
S_{y p}\left(z^{-1}\right)-S_{y b}\left(\mathrm{z}^{-1}\right)=1
$$

\section{DEFINITION OF A 'TEMPLATE' FOR THE OUTPUT SENSITIVITY FUNCTION}

Using the small gain theorem and various representations of the plant uncertainties, the modulus margin and the delay margin can be converted into robust stability conditions. On the other hand, the robust stability conditions allow the definition of upper templates for the modulus of the various sensitivity functions. ${ }^{1,9,10}$

For a delay margin of one sampling period the robust stability condition is expressed as follows: ${ }^{11}$

$$
\left|S_{y b}\left(z^{-1}\right)\right|<\left|\left(1-z^{-1}\right)\right|^{-1}, \quad z=e^{-j \omega}, \quad 0 \leqslant \omega \leqslant \pi
$$

But, from equation (11) we have

$$
1-\left|S_{y b}\left(z^{-1}\right)\right|<\left|S_{y p}\left(z^{-1}\right)\right|<1+\left|S_{y b}\left(z^{-1}\right)\right|
$$

If $S_{y b}\left(z^{-1}\right)$ satisfies the condition (12) then $S_{y p}\left(z^{-1}\right)$ will satisfy the following condition:

$$
1-\left|1-z^{-1}\right|^{-1}<\left|S_{y p}\left(z^{-1}\right)\right|<1+\left|1-z^{-1}\right|^{-1} ; \quad z=e^{-j \omega} ; \quad 0 \leqslant \omega \leqslant \pi
$$

Therefore in order to assure the delay margin $\Delta \tau=T_{s}$, it is required that the modulus of $S_{y p}\left(\mathrm{z}^{-1}\right)$ lies between the bounds defined by a lower template $\left|W^{-1}\right|_{\text {inf }}=1-\left|1-z^{-1}\right|^{-1}$ and an upper template defined by $\left|W^{-1}\right|_{\text {sup }}=1+\left|1-z^{-1}\right|^{-1}$.

The nominal performance requirements and the robust stability conditions lead to the definition of a desired template for the sensitivity function. We will subsequently consider the definition of such a template for the output sensitivity function. The desired template takes, in general, the form shown in Figure 2. Regarding the robust stability, the chosen modulus margin will define the maximum value of the modulus of the output sensitivity function (upper template) and the chosen delay margin will define an upper and a lower template starting, for example, around $0.15 f_{s}$ (for $\left.\Delta \tau=T_{s}\right)$. 


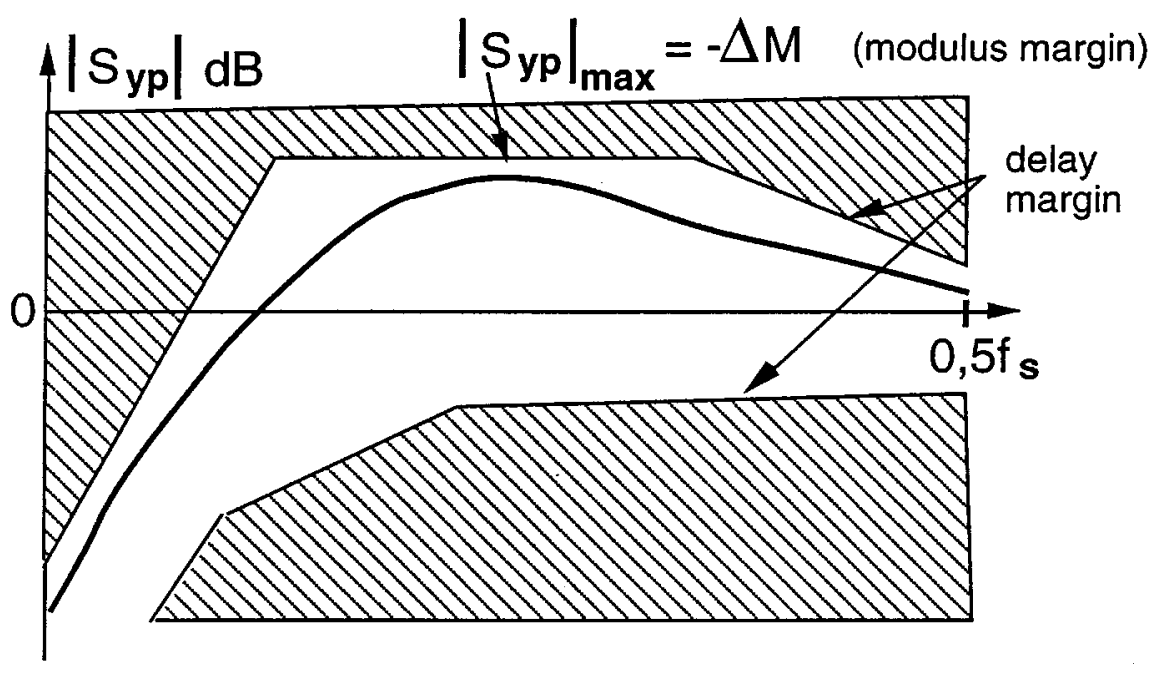

Figure 2. Desired template for the output sensitivity function (the case of low frequencies disturbance rejection)

A template for the input sensitivity function also may be defined in the same way. Additive uncertainty can be used as an upper template to assure the robust stability of the closed-loop system. Another upper bound should be considered for the frequencies where the plant gain is very low, usually in the high frequencies and at the frequencies of the low damped zeros of the plant model, in order to prevent the unwanted excitation of control input which has no effect in the output.

\section{METHOD A}

\subsection{Pole placement}

In this section the pole placement technique is reviewed; for more details see Reference 12 . The dominant closed-loop poles are chosen to satisfy the desired regulation performance. The auxiliary poles can be introduced either for filtering effects in certain frequency regions or for reducing the stress on the actuator as well as for improving the robustness of the closed-loop system (as will be shown subsequently).

For different reasons, i.e., disturbance rejection, signal blocking, etc. the polynomials $R\left(z^{-1}\right)$ and $S\left(z^{-1}\right)$ generally contain some fixed parts which are specified before solving equation (7) (for example the polynomial $S\left(z^{-1}\right)$ should contain a factor $\left(1-z^{-1}\right)$ for a null steady state error). In order to take into account these prespecified parts, the polynomials $R\left(z^{-1}\right)$ and $S\left(z^{-1}\right)$ are factored as:

$$
\begin{aligned}
& R\left(z^{-1}\right)=R^{\prime}\left(z^{-1}\right) \cdot H_{R}\left(z^{-1}\right) \\
& S\left(z^{-1}\right)=S^{\prime}\left(z^{-1}\right) \cdot H_{S}\left(z^{-1}\right)
\end{aligned}
$$

where $H_{R}\left(z^{-1}\right)$ and $H_{S}\left(z^{-1}\right)$ are the prespecified polynomials. The closed-loop poles in equation (7) become

$$
P\left(z^{-1}\right)=A\left(z^{-1}\right) \cdot H_{S}\left(z^{-1}\right) \cdot S^{\prime}\left(z^{-1}\right)+z^{-d} B\left(z^{-1}\right) \cdot H_{R}\left(z^{-1}\right) \cdot R^{\prime}\left(z^{-1}\right)
$$


The design procedure can be summarized as follows:

(a) Choose the desired closed-loop poles $P\left(z^{-1}\right)$, the fixed parts of the controller $H_{R}\left(z^{-1}\right)$, $H_{S}\left(\mathrm{z}^{-1}\right)$ and the desired tracking dynamics $B_{m}\left(z^{-1}\right) / A_{m}\left(z^{-1}\right)$.

(b) Compute $S^{\prime}\left(z^{-1}\right)$ and $R^{\prime}\left(z^{-1}\right)$ by solving equation (17) and consequently compute $S\left(z^{-1}\right)$ and $R\left(z^{-1}\right)$ using equations (15) and (16).

(c) Compute the prefilter $T\left(z^{-1}\right)=P\left(z^{-1}\right) / B(1)$. This choice leads to the following transfer function between the reference and the plant output:

$$
H_{y r}\left(z^{-1}\right)=\frac{z^{-d} B_{m}\left(z^{-1}\right)}{A_{m}\left(z^{-1}\right)} \cdot \frac{B\left(z^{-1}\right)}{B(1)}
$$

Another method consists of taking $T\left(z^{-1}\right)=R(1)$.

$$
H_{y r}\left(z^{-1}\right)=\frac{z^{-d} B_{m}\left(z^{-1}\right)}{A_{m}\left(z^{-1}\right)} \cdot \frac{B\left(z^{-1}\right)}{P\left(z^{-1}\right)} \cdot \frac{P(1)}{B(1)}
$$

Of course intermediate solutions between (18)- and (19)- can also be obtained, that is, to choose $T\left(z^{-1}\right)$ such that it compensates only a part of the poles of $P\left(z^{-1}\right)$.

\subsection{Shaping the sensitivity functions}

The dominant poles $P_{D}\left(z^{-1}\right)$, the auxiliary poles $P_{F}\left(z^{-1}\right)$ and the two filters $H_{R}\left(z^{-1}\right)$ and $H_{S}\left(z^{-1}\right)$ are the tools for shaping the sensitivity functions in order to meet the specifications defined by the desired templates. Placing a pair of complex zeros in $H_{S}\left(\mathrm{z}^{-1}\right)$ or in $H_{R}\left(\mathrm{z}^{-1}\right)$ decreases the magnitude of the modulus of the sensitivity functions around the frequencies of these zeros. Introducing a pair of pure imaginary zeros at a certain frequency in $H_{R}\left(z^{-1}\right)$ will make $\left|S_{y p}\right|=1$ and $\left|S_{u p}\right|=0$ at this frequency. Complex zeros will bring $\left|S_{y p}\right|$ closer to the $0 \mathrm{~dB}$ axis. Putting some asymptotically stable real high frequency poles in $P\left(\mathrm{z}^{-1}\right)$ will cause an attenuation of $\left|S_{y p}\right|$ in the domain of attenuation of $1 / P_{F}\left(z^{-1}\right)$. Placing a pair of complex poles at a certain frequency in $P_{F}\left(z^{-1}\right)$ will lead to the increase of $\left|S_{y p}\right|$ around this frequency. Introducing a term of $\left(1+z^{-1}\right)$ in $H_{R}\left(z^{-1}\right)$ will open the loop in the high frequencies.

The underlying philosophy of the design is to choose the closed-loop poles and the fixed parts of the controller in order to bring the sensitivity functions within the defined templates. This controller will meet the conditions on modulus and delay margin as well as the attenuation band and will assure the robust stability of the closed-loop system against the parameter uncertainty. The shaping method which is an iterative procedure (generally converging in few steps) is as follows:

\section{Step I}

- Choose the dominant closed-loop poles of $P\left(z^{-1}\right)$ and the fixed part of $R\left(z^{-1}\right)$ and $S\left(z^{-1}\right)$ in order to meet the nominal performance specifications.

- Compute the controller.

- Check the shape of the output sensitivity function. If the upper bound of the template is not satisfied, generally two situations are identified:

(a) the maximum of the output sensitivity function is located in the frequency range next to the attenuation band. In this case, go to step II;

(b) the maximum of the output sensitivity function is located in the high frequency range (near $0 \cdot 5 f_{s}$ ). In this case jump to step III. 
Step II

- Add a pair of complex zeros to $H_{S}\left(z^{-1}\right)$ :

$$
H_{S}\left(z^{-1}\right)=1+\alpha_{1} z^{-1}+\alpha_{2} z^{-2}
$$

The frequency of these zeros is chosen close to the frequency where the maximum of the $\left|S_{y p}\right|$ occurs (between the maximum frequency of the attenuation band and the frequency where $\left|S_{y p}\right|_{\max }$ occurs). The damping factor is chosen such that the introduced attenuation brings $\left|S_{y p}\right|_{\text {max }}$ below the admissible value. Typical values are between $\zeta=0 \cdot 3$ to $0 \cdot 8$.

- Recompute the controller. If $\left|S_{y p}\right|$ is too large in the high frequency range, go to step III.

\section{Remark}

The effects of these additional zeros are in general:

(a) a reduction in the modulus of the sensitivity function in the region next to the attenuation band;

(b) an increase in the maximum of the modulus of the sensitivity function in the high frequency range (in general its maximum is shifted in the high frequency range);

(c) an increase in the attenuation band.

\section{Step III}

- Add auxiliary high frequency poles of the form:

$$
P_{F}\left(z^{-1}\right)=\left(1-p_{1} z^{-1}\right)^{n_{F}} ; \quad 0.05 \leqslant p_{1} \leqslant 0 \cdot 5
$$

where

$$
n_{F} \leqslant n_{P}-\mathrm{n}_{D} ; \quad n_{P}=(\operatorname{deg} P)_{\max } ; \quad n_{D}=\operatorname{deg} P_{D}
$$

with increasing values of $p_{1}$ starting from 0.05 till the specifications on the $\left|S_{y p}\right|$ in the high frequency range are met. It should be mentioned that high frequency auxiliary poles shift, in general, the maximum of the $\left|S_{y p}\right|$ towards the lower frequency range. If the $\left|S_{y p}\right|_{\max }$ is satisfactory, stop here, if not go to step IV.

Step IV This step is similar to step II

- If the new maximum is close to the one resulting after step I, change accordingly the frequency and the damping of the zeros introduced in $H_{S}$.

- If the new maximum is at a significantly different frequency, add a new pair of complex zeros in $H_{S}\left(z^{-1}\right)$ at a frequency close to the maximum of $\left|S_{y p}\right|$. The damping is chosen in order to bring $\left|S_{y p}\right|_{\max }$ below the maximum acceptable value. If the results are unsatisfactory, go to step V.

Step $V$

- The value of the real auxiliary poles (defined in step III) can be increased.

- If necessary, the dominant poles may also be slowed down.

- End of the procedure for the shaping of the output sensitivity function. Go to step VI.

\section{Step VI}

- Check the input sensitivity function. Add, if necessary, high frequency complex zeros in $H_{R}\left(z^{-1}\right)$ (often at $0 \cdot 5 f_{s}$ ). 
- Check again the output sensitivity function. Three different situations may occur:

(a) the output sensitivity function is over the template in the high frequencies. Go to step V;

(b) the output sensitivity function is over the template in the low frequencies. Go to step IV;

(c) the output sensitivity function is inside the template. End of the procedure.

The flowchart of this procedure is illustrated in Figure 3.

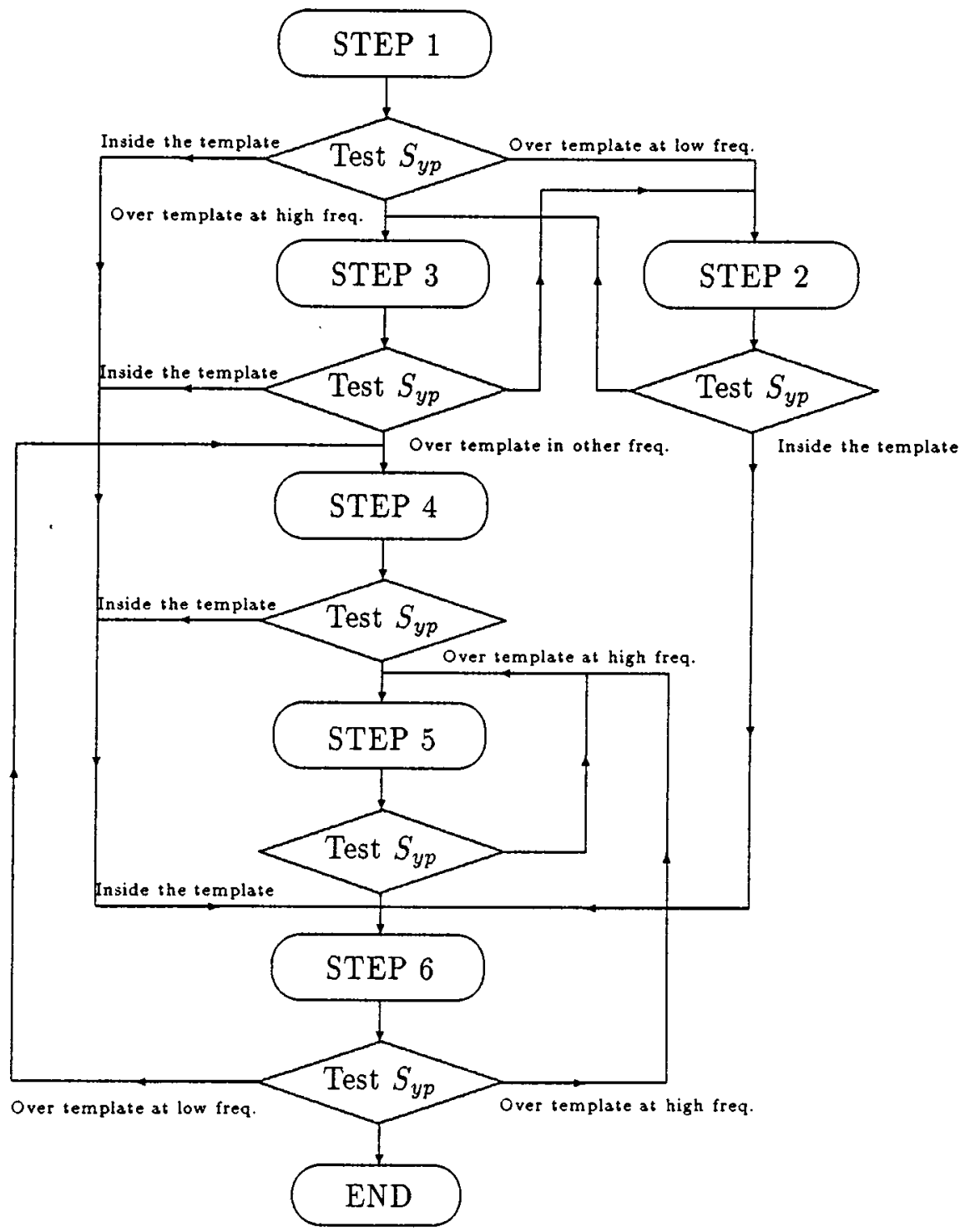

Figure 3. The flow chart of the iterative procedure of Method A 


\section{METHOD B}

The problem is to find a controller which guarantees the internal stability of the closed loop, assures desired dominant closed-loop poles and satisfies certain constraints on the output sensitivity function. To solve this problem, first a desired sensitivity function which satisfies the constraints on the output sensitivity function is computed. The use of an appropriate fixed part in the desired sensitivity function will assure a partial pole placement to desired values. Then the inverse of the desired sensitivity function will be used as a weighting filter to minimize the infinity norm of the output sensitivity function. Therefore, the real output sensitivity function will approximate the desired one. The internal stability of the closed loop will be guaranteed by using the Youla parametrization in the $H_{\infty}$ optimization.

The demonstration will be carried out in discrete time but it could be done for continuous time as well.

\subsection{Desired sensitivity function}

It was shown in Section 3 that the output sensitivity function may be written in the following form:

$$
S_{y p}\left(z^{-1}\right)=\frac{A\left(z^{-1}\right) S\left(z^{-1}\right)}{A\left(z^{-1}\right) S\left(z^{-1}\right)+z^{-d} B\left(z^{-1}\right) R\left(z^{-1}\right)}=\frac{A\left(z^{-1}\right) S\left(z^{-1}\right)}{P_{D}\left(z^{-1}\right) P_{F}\left(z^{-1}\right)}
$$

Where $P_{D}$ and $P_{F}$ are the dominant and auxiliary poles of the closed loop. Since $A$ and the desired closed-loop poles $P_{D}$ are known, we can fix them in the desired sensitivity function. Therefore we can define

$$
S_{d}\left(z^{-1}\right)=\frac{A\left(z^{-1}\right)}{P_{D}\left(z^{-1}\right)} \cdot \frac{X\left(z^{-1}\right)}{Y\left(z^{-1}\right)}
$$

where $S_{d}$ is the desired sensitivity function and the polynomials $X$ and $Y$ should be computed using an optimization method.

It can be proved ${ }^{7}$ that the denominator of $S_{d}$ will appear as a part of the final closed-loop poles if the inverse of $S_{d}$ is utilized as a weighting filter in the $H_{\infty}$ optimization method. This technique, known as partial pole placement, ${ }^{7,8}$ rejustifies the interpretation of the weighting filter as the inverse of the desired sensitivity function. On the other hand, natural choice of the polynomial $A$ in the numerator of $S_{d}$ will prevent the presence of the open-loop poles as closed-loop poles. ${ }^{7}$

In the next step, the polynomials $X$ and $Y$ will be parametrized. They are parametrized preferably as products of real zeros and poles, respectively, in order firstly to prevent the unwanted cancellation of the desired poles $P_{D}$ or of the open-loop poles $A$ which may contain low damped complex poles, and secondly to avoid the oscillatory poles in the polynomial $Y$ which will appear consequently as the closed-loop poles.

Then the polynomials $X$ and $Y$ are defined as follows:

$$
\begin{aligned}
X\left(z^{-1}\right) & =\left(1-x_{1} z^{-1}\right)\left(1-x_{2} z^{-1}\right) \cdots\left(1-x_{n_{X}} z^{-1}\right) \\
Y\left(z^{-1}\right) & =\left(1-y_{1} z^{-1}\right)\left(1-y_{2} z^{-1}\right) \cdots\left(1-y_{n_{Y}} z^{-1}\right) \\
\theta & =\left[x_{1}, x_{2}, \ldots, x_{n_{X}}, y_{1}, y_{2}, \ldots, y_{n_{Y}}\right]
\end{aligned}
$$

where $x_{1}$ to $x_{n_{X}}$ are the real zeros of $X, y_{1}$ to $y_{n_{Y}}$ are the real zeros of $Y$ and $\theta$ is the vector of the parameters. It may be considered that $S_{d}\left(z^{-1}, \theta\right)$ is proper and inversely proper, i.e., the order of the numerator is equal to the order of denominator. This leads to a proper weighting filter for 
$H_{\infty}$ optimization. It should be mentioned that the properness of the weighting filter can be relaxed. ${ }^{13}$

It can be shown that the denominator of the weighting filter will appear as a part of the denominator of the controller. Then, in this approach, the desired fixed parts in the denominator of the controller can be put directly in the numerator of the $S_{d}$. Thus, for example, to reject the constant disturbances in the output, an integrator may be used in $S_{d}\left(z^{-1}, \theta\right)$. This can be easily done by choosing $x_{1}=1$.

The minimum order of $X$ and $Y$ depends on the order of $A, P_{D}$ and the fixed parts of $Y$. We have

$$
n_{X} \geqslant n_{A}+\left(n_{Y}\right)_{\min }-n_{P_{D}}
$$

where $n_{A}, n_{X}, n_{P_{D}}$ and $\left(n_{Y}\right)_{\min }$ are the orders of $A, X, P_{D}$ and the fixed parts of $Y$, respectively. For example, if we choose $\mathrm{n}_{P_{D}}=n_{A}$ and an integrator in $Y$ we will obtain $n_{X}=n_{Y}=1$. In this case we have only to compute one parameter $x_{1}$. If with this parameter we do not succeed in minimizing the criterion (which will be explained subsequently) and in satisfying the constraints, we can increase the order of $X$ and $Y$ to have more degrees of freedom in the optimization program.

\subsection{Performance index}

The unstable poles and zeros of the open loop, i.e., the poles and zeros outside the unit circle in discrete time or with a positive real part in continuous time, play an important role in the performance of the closed loop and consequently in the form of the sensitivity function. Therefore, these unstable poles and zeros should be considered in the design of a desired sensitivity function. A central result, which explains the relation between the unstable poles and zeros of the open loop and the sensitivity function, is the Zames-Francis equality, ${ }^{5,6}$ which is based on the Poisson integral formula from complex function theory. The result that follows is the discrete-time version of this equality which was obtained by Sung and Hara. ${ }^{14}$

Suppose that $\beta=r e^{j \phi}$ is an unstable zero of the open loop. If the closed-loop system is stable, then the following relation holds:

$$
\pi \log \left|B_{\alpha}^{-1}(\beta)\right|=\int_{-\pi}^{\pi} \log \left|S_{y p}(j \omega)\right| \cdot \frac{r^{2}-1}{1-2 r \cos (\omega-\phi)+r^{2}} \cdot d \omega
$$

where $B_{\alpha}(\beta)$ is the Blaschke product

$$
B_{\alpha}(z)=\prod_{i} \frac{z-\alpha_{i}}{1-\bar{\alpha}_{i} z}
$$

formed from the unstable poles of the open loop $\alpha_{i}$ (here the overbar denotes the complex conjugate). Since this equality is satisfied by $S_{y p}$, it should also be satisfied by the desired sensitivity function $S_{d}\left(z^{-1}, \theta\right)$. Therefore, we can choose this equality as a criterion for the optimization program in order to find the polynomials $X$ and $Y$. The criterion can be expressed as a function of $\theta$ (the parameter vector) as follow:

$$
J_{i}(\theta)=\left|\int_{-\pi}^{\pi} \log \right| S_{d}(j \omega, \theta)\left|\cdot \frac{r_{i}^{2}-1}{1-2 r_{i} \cos \left(\omega-\phi_{i}\right)+r_{i}^{2}} \cdot d \omega-\pi \log \right| B_{\alpha}^{-1}\left(\beta_{i}\right)||
$$

where $\beta_{i}=r_{i} e^{j \phi_{i}}$ are the unstable zeros of the open loop. In fact, only the unstable zeros of the plant are known so the number of criteria is equal to the number of the unstable zeros of the plant. It should be mentioned that a time delay in the discrete-time system is in fact an unstable 
zero at infinity, that means $r$ goes to infinity in equation (29) and the criterion becomes:

$$
J_{d}(\theta)=\left|\int_{-\pi}^{\pi} \log \right| S_{d}(j \omega, \theta)\left|d \omega-\pi \sum_{i} \log \right| \alpha_{i}||
$$

The objective will be then to minimize the multi-objective function:

$$
J(\theta)=\left[J_{1}(\theta), J_{2}(\theta), \ldots, J_{n_{u z}}(\theta), J_{d}(\theta)\right]
$$

where $n_{u z}$ is the number of the unstable zeros of the plant. It is of course very difficult to minimize all these criteria simultaneously. An alternative might be to minimize the maximum value of $J(\theta)$.

\section{Remark}

The second term in the criterion $J_{i}(\theta)$ is related to the unstable poles of the open loop. Consider that the plant is strongly stabilizable, then it is possible to compute a stable controller which will stabilize the closed loop. Thus, it may be supposed that the unstable poles of the open loop are the only poles of the plant, if there are any. With this hypothesis we search the stable controllers among all of the stabilizing controllers which satisfy the constraints. Then this hypothesis will be verified by the final controller if the $H_{\infty}$ norm of the output sensitivity function filtered by the inverse of $S_{d}$ is one, i.e., $\left\|S_{d}^{-1} \cdot S_{y p}\right\|_{\infty}=1$.

\subsection{Constraints}

It was shown that the robustness margins, i.e., modulus and delay margin, and the model uncertainty, as well as the performance of the closed-loop system for output disturbance rejection, can be defined as a template in the frequency domain for the output sensitivity function. This template then, may be used as the constraint in the optimization algorithm to compute the desired sensitivity function. These constraints are expressed as follows:

$$
C_{L}(\omega) \leqslant\left|S_{d}(j \omega, \theta)\right| \leqslant C_{U}(\omega)
$$

where $C_{U}(\omega)$ and $C_{L}(\omega)$ are the upper and lower bound for the output sensitivity function, respectively.

\subsection{Optimization program}

The optimization program can be summarized as follows:

- Definition of the structure of the desired sensitivity function and the vector of parameters

$$
\begin{gathered}
S_{d}\left(z^{-1}\right)=\frac{A\left(z^{-1}\right)}{P_{D}\left(z^{-1}\right)} \cdot \frac{\left(1-x_{1} z^{-1}\right)\left(1-x_{2} z^{-1}\right) \ldots\left(1-x_{n_{x}} z^{-1}\right)}{\left(1-y_{1} z^{-1}\right)\left(1-y_{2} z^{-1}\right) \ldots\left(1-y_{n_{Y}} z^{-1}\right)} \\
\theta=\left[x_{1}, x_{2}, \ldots, x_{n_{x}}, y_{1}, y_{2}, \ldots, y_{n_{Y}}\right]
\end{gathered}
$$

- Definition of the performance index according to the Zames-Francis equality

$$
\begin{gathered}
J_{i}(\theta)=\left|\int_{-\pi}^{\pi} \log \right| S_{d}(j \omega, \theta)\left|\cdot \frac{r_{i}^{2}-1}{1-2 r_{i} \cos \left(\omega-\phi_{i}\right)+r_{i}^{2}} \cdot d \omega-\pi \log \right| B_{\alpha}^{-1}\left(\beta_{i}\right)|| \\
\theta=\operatorname{Arg} \min _{\theta} \max _{i} J_{i}(\theta)
\end{gathered}
$$


- Definition of the upper and lower bound for the desired sensitivity function

$$
C_{L}(\omega) \leqslant\left|S_{d}(j \omega, \theta)\right| \leqslant C_{U}(\omega)
$$

If the results of the optimization program are not satisfactory in terms of the constraints or the performance index, we can increase the number of parameters, i.e., the order of $X$ and $Y$, or eventually we may use a pair of complex poles and zeros in $S_{d}$. However, the experimental results show that even for complex systems it is not necessary to use complex poles and zeros in $S_{d}$. This optimization problem can be solved using the CONSTR instruction in the optimization toolbox of MATLAB.

\section{5. $H_{\infty}$ optimization}

After computing the desired sensitivity function $S_{d}\left(z^{-1}\right)$, the output sensitivity function $S_{y p}\left(z^{-1}\right)$ filtered by the inverse of $S_{d}\left(z^{-1}\right)$ will be minimized using the $H_{\infty}$ optimization method. This problem, known as the model matching problem, is the simplest problem in the $H_{\infty}$ domain. This problem may be solved either iteratively by the standard $H_{\infty}$ optimal control problem using the $\mu$-synthesis toolbox in MATLAB or directly by Nevanlinna-Pick's algorithm. ${ }^{9}$ The second method is more suitable for this technique since in the model matching problem the optimal solution can be achieved without any iteration. Therefore, a discrete time version of the Nevanlinna-Pick algorithm has been developed to solve the application problem of Section 7.

It should be noticed that in this technique only the partial pole placement, i.e., the closed-loop poles defined by $P_{D}$, is guaranteed, so it is possible to obtain the other closed-loop poles near to the unit circle. To avoid this problem we can change the stability domain by choosing a circle with a radius less than one (or axis shifting in continuous-time domain).

It was mentioned in Section 4 that we need usually to open the loop or to reduce drastically the gain of the polynomial $R$ at certain frequencies where the gain of the system is very low in order to prevent the unwanted excitation in the control input. It means that it is necessary to shape the input sensitivity function simultaneously with the output sensitivity function, which is called the mixed sensitivity problem in the literature. ${ }^{10}$ An alternative way is to augment the order of the terms of $B$ (the numerator of the plant) by introducing terms of the form $\left(1+\alpha z^{-1}\right)$ with $\alpha>0.7$ which places zeros at $0 \cdot 5 f_{s}$ and of the form $\left(1+\alpha_{1} z^{-1}+\alpha_{2} z^{-2}\right)$ which introduce complex zeros at other desired frequencies. Choosing an appropriate damping factor for the complex zeros will reduce significantly $S_{u p}$ at the corresponding frequencies. These terms correspond to roots which are very close to the unit circle and will be moved back to the controller (polynomial $R$ ) after computing it. It should be noticed that the stable zeros of the plant will be cancelled by the controller in the $\mathrm{H}_{\infty}$ approach. Then, in order to avoid the cancellation of these zeros, they should appear as unstable zeros. This is achieved by defining a reduced stability domain, leaving these roots outside. Using this technique we can shape two sensitivity functions by only one weighting filter.

\section{APPLICATIONS}

In this section a flexible transmission system which has been the subject of two benchmarks on adaptive control ${ }^{15}$ and on robust digital control ${ }^{16}$ and has been also used as an example by Kwakernaak ${ }^{17}$ is considered. For this system three models of the plant and several specifications are given. 


\subsection{System description}

The flexible transmission consists of three horizontal pulleys connected by two elastic belts (Figure 4). The first pulley is driven by a DC motor whose position is controlled by local feedback. The third pulley may be loaded by small disks of different weights. The system input is the reference for the axis position of the first pulley. The system output is the axis position of the third pulley measured by a position sensor, sampled and digitized. A PC is used to control the system. The main characteristics of this system are the low damped vibration modes (damping factor less than 0.05) and the large variations of their frequencies with the load. The discrete-time identified models $\left(T_{s}=50 \mathrm{~ms}\right.$ ) of the plant for two extreme situations (no load and full load), as well as for an intermediate situation are given.

Unloaded model:

$$
\begin{aligned}
& A_{0}\left(q^{-1}\right)=1-1 \cdot 41833 q^{-1}+1 \cdot 58939 q^{-2}-1 \cdot 31608 q^{-3}+0 \cdot 88642 q^{-4} \\
& B_{0}\left(q^{-1}\right)=0 \cdot 28261 q^{-1}+0.50666 q^{-2} ; \quad d=2
\end{aligned}
$$

Half load model:

$$
\begin{aligned}
& A_{1}\left(q^{-1}\right)=1-1 \cdot 99185 q^{-1}+2 \cdot 20265 q^{-2}-1 \cdot 84083 q^{-3}+0 \cdot 89413 q^{-4} \\
& B_{1}\left(q^{-1}\right)=0 \cdot 10276 q^{-1}+0 \cdot 18123 q^{-2} ; \quad d=2
\end{aligned}
$$

Full load model:

$$
\begin{aligned}
& A_{2}\left(q^{-1}\right)=1-2 \cdot 0967 q^{-1}+2 \cdot 3196 q^{-2}-1.93353 q^{-3}+0 \cdot 87129 q^{-4} \\
& B_{2}\left(q^{-1}\right)=0 \cdot 06408 q^{-1}+0 \cdot 10407 q^{-2} ; \quad d=2
\end{aligned}
$$

Several time and frequency specifications are considered for this problem which should be satisfied for all loads:

(a) A rise time $(0-90 \%$ of the final value) of less than $1 \mathrm{~s}$ for a step change in the reference input.

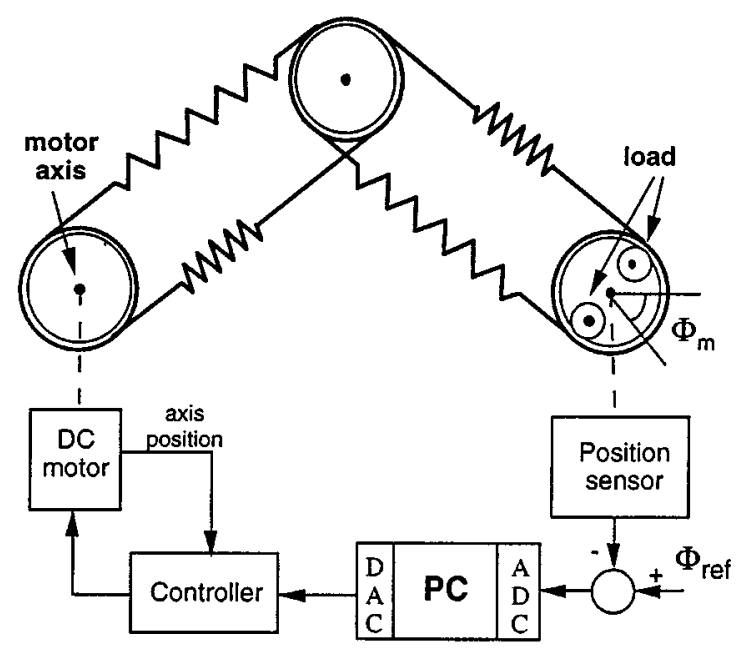

Figure 4. Schematic diagram of the flexible transmission 
(b) Overshoot less than $10 \%$ for a step change in the reference input.

(c) Rejection of step output disturbances filtered by $1 / A$ within $1 \cdot 2 \mathrm{~s}$ (for $90 \%$ rejection of the measured peak value).

(d) Perfect rejection of constant disturbances (using integral action).

(e) Disturbance attenuation in the low frequency band from 0 to $0 \cdot 2 \mathrm{~Hz}$.

(f) A maximum value of less than $6 \mathrm{~dB}$ of the output sensitivity function (modulus margin greater than $0 \cdot 5)$.

(g) A delay margin of at least $40 \mathrm{~ms}(80 \%$ of sampling period).

(h) A maximum value of less than $10 \mathrm{~dB}$ of the input sensitivity function in the frequency range $8-10 \mathrm{~Hz}$.

For more details see Reference 16.

\subsection{Controller design}

A complete solution to this flexible transmission problem by Method A has been given in Reference 3. This problem will be redesigned by Method B in the following steps:

1. The unloaded model which seems to be the most difficult to control is chosen as the nominal model.

2. Two pairs of complex poles, with the same frequencies as those of the vibration modes of the nominal plant, but with a damping factor of 0.8 for the first mode (which is more energetic) and of $0 \cdot 2$ for the second mode, are considered as the dominant closed-loop poles.

3. The desired sensitivity function is defined as follows:

$$
S_{d}\left(z^{-1}\right)=\frac{A_{0}\left(z^{-1}\right)}{P_{D}\left(z^{-1}\right)} \cdot \frac{\left(1-x_{1} z^{-1}\right)\left(1-x_{2} z^{-1}\right)}{\left(1-y_{1} z^{-1}\right)\left(1-y_{2} z^{-1}\right)}
$$

4. In order to obtain an integrator in the controller, $x_{1}$ should be equal to one. This means that the weighting filter will have a pole on the unit circle, which is not allowed in $H_{\infty}$ optimization. To avoid this problem we can choose $x_{1}=0.999999$.

5. It remains to compute three parameters in $S_{d}$ by the optimization program. Therefore, the parameter vector $\theta$ will be: $\theta=\left[x_{2}, y_{1}, y_{2}\right]$.

6. The nominal model has an unstable zero and a time delay, therefore the performance index is defined as $J(\theta)=\max \left[J_{1}(\theta), J_{d}(\theta)\right]$ where $J_{1}$ and $J_{d}$ correspond to the unstable zero and the time delay in equations (31) and (32), respectively.

7. A template, like the template in Figure 2, with a minimum modulus margin equal to $0 \cdot 5$ and a delay margin of one sampling period, is chosen for the desired output sensitivity function.

8. A constrained optimization program is run to find the vector of parameters. The results of the optimization program are $x_{2}=-0.3986, y_{1}=0.95$ and $y_{2}=0.4907$. This solution leads to the following weighting filter:

$$
W\left(z^{-1}\right)=S_{d}^{-1}\left(z^{-1}\right)=\frac{P_{D}\left(1-0 \cdot 95 z^{-1}\right)\left(1-0 \cdot 4907 z^{-1}\right)}{A_{0}\left(z^{-1}\right)\left(1-0 \cdot 999999 z^{-1}\right)\left(1+0 \cdot 3986 z^{-1}\right)}
$$

where

$$
P_{D}=\left(1-1 \cdot 1396 z^{-1}+0 \cdot 3734 z^{-2}\right)\left(1+0 \cdot 0886 z^{-1}+0 \cdot 5135 z^{-2}\right)
$$


9. The stability domain is reduced to a circle with radius 0.85 centred at $\alpha=0 \cdot 15$. Then, the plant model and weighting filter equations are transformed accordingly to this new stability domain (an $r$ is used for the parameter in the new stability domain, for example, $W r\left(z^{-1}\right)$ denotes the weighting filter in the reduced stability domain). It should be noticed that the reduced stability domain must contain the point $[1,0 j]$ in order to keep the integrator in the weighting filter.

10. The weighting filter in the new system, which may have unstable poles, is factorized into an all-pass function and a stable filter which will be used in $H_{\infty}$ optimization.

11. The infinity norm of $\operatorname{Wr}\left(z^{-1}\right) S r_{y p}\left(z^{-1}\right)$ should be minimized. First of all, using the $Q$ parametrization $S r_{y p}$ is written as follows:

$$
S r_{y p}\left(z^{-1}\right)=1-\frac{B r_{0}\left(z^{-1}\right)}{A r_{0}\left(z^{-1}\right)} Q\left(z^{-1}\right)
$$

then, this problem is converted to the following model matching problem:

$$
\min \left\|T_{1}-T_{2} Q\right\|_{\infty}=\gamma
$$

where $T_{1}=W r$ and $T_{2}=W r \cdot B r_{0} / A r_{0}$. The optimum value of $\gamma$ is computed using the Nehari's theorem. ${ }^{18}$ Next, the parameters of $Q$ are calculated using coprime factorization and Nevanlinna-Pick's algorithm in discrete time. Finally, the controller parameters are determined using the following relation:

$$
\frac{R r}{S r}=\frac{Q}{1-Q B r_{0} / A r_{0}}
$$

where $\mathrm{Rr} / \mathrm{Sr}$ is the controller in the reduced stability domain.

12. The controller is transferred back to the real stability domain and its order is reduced by zero/pole cancellation techniques.

Although this controller satisfies the robustness margins, places the closed-loop poles in the desired places and stabilizes two other plant models, it is still far from some of the specifications of the benchmark problem. In order to improve the sensitivity function for the other plants, we can reduce the desired output sensitivity function in the frequency region where the maximum parameter uncertainty occurs. This region corresponds to the maximum of additive uncertainty and is located between the frequency of the first vibration mode of the full loaded model and that of the unloaded one. Introducing two pairs of complex zeros in this frequency region with an appropriate damping factor as a fixed part in the numerator of the desired sensitivity function leads to reducing the output and input sensitivity function in the critical region. Figure 5 shows the desired sensitivity function (inverse of the weighting filter) before (dashed line) and after introducing the following fixed part:

$$
H_{S}\left(z^{-1}\right)=\left(1-1.7052 z^{-1}+0.7753 z^{-2}\right)\left(1-1.4976 z^{-1}+0.6337 z^{-2}\right)
$$

corresponding to $\left(\omega_{0}=5 \cdot 65, \zeta=0 \cdot 45\right)$ and $\left(\omega_{0}=8 \cdot 29, \zeta=0 \cdot 55\right)$.

Figures 6 and 7 show the output and input sensitivity functions, respectively, for the three models using the following simplified controller:

$$
\begin{aligned}
& R\left(z^{-1}\right)=0 \cdot 4075-1 \cdot 2444 z^{-1}+0 \cdot 8792 z^{-1} \\
& S\left(z^{-1}\right)=1-0 \cdot 5386 z^{-1}-0 \cdot 4614 z^{-2} \\
& T\left(z^{-1}\right)=0 \cdot 4572-0 \cdot 3232 z^{-1}-0 \cdot 0912 z^{-2}
\end{aligned}
$$




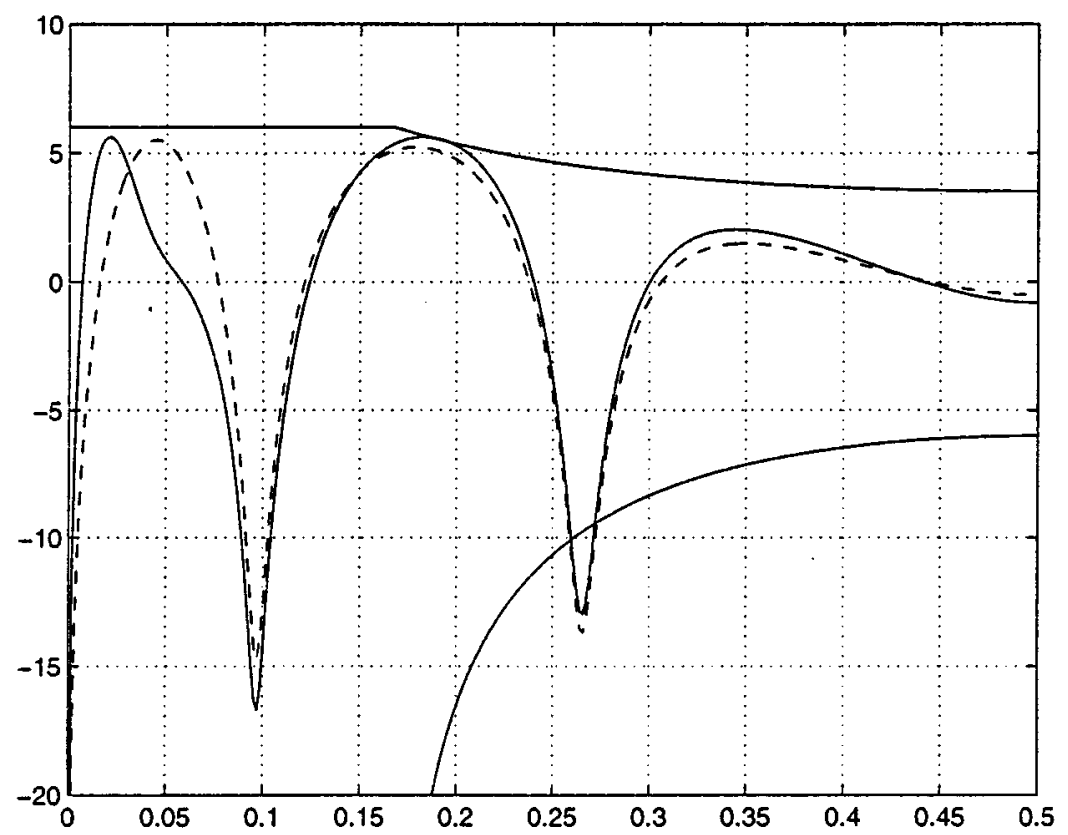

Figure 5. Desired sensitivity function

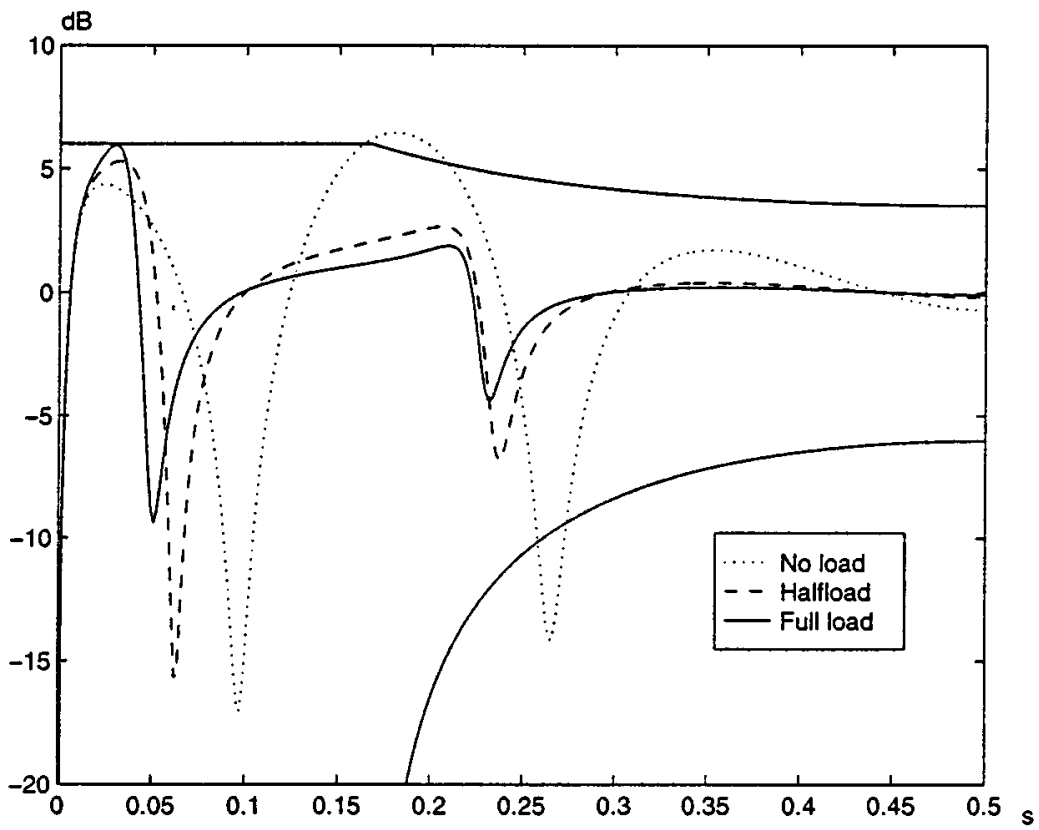

Figure 6 . The output sensitivity functions for the different loadings 


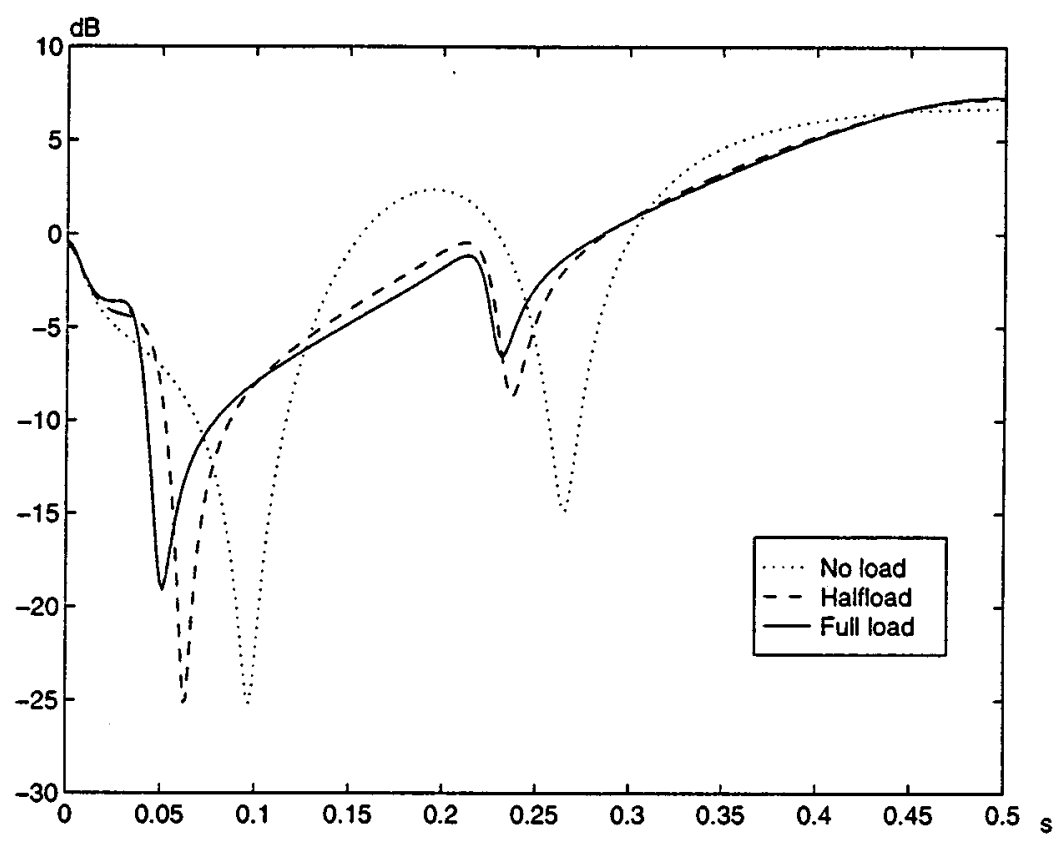

Figure 7. The input sensitivity functions for the different loadings

A second order system with $\omega_{0}=6 \cdot 3$ (which corresponds to the first vibration mode of the full load model) and $\zeta=0.8$ is chosen for the tracking reference model. The parameters of the prefilter $T\left(z^{-1}\right)$ are computed independently from the controller. In fact the parameters of $T\left(z^{-1}\right)$ are the results of an optimization program which minimizes the overshoot and rise time for the three models. Figures 8 and 9 show the step and disturbance (filtered by $1 / A$ ) responses for the three models.

Tables 1 and 2 show the simulation and experimental results of the controller in the different loadings, respectively.

This controller satisfies $85 \%$ of the specifications of the benchmark in simulation. A comparison, in terms of satisfaction of performances and complexity with the controller designed by Method A, the $H_{\infty}$ controllers in the benchmark designed by Jones and Limebeer ${ }^{19}$ and Walker, ${ }^{20}$ and the controller designed by Kwakernaak, ${ }^{17}$ is presented in Table 3. For a real time comparison, only the time characteristics, i.e., rise time, settling time and disturbance rejection time, are available. Therefore the normalized sum (with respect to the desired values) of these characteristics for the three cases is used as a performance criterion (smaller criterion means better performance). This criterion is then penalized by the normalized complexity of the controller (defined as the sum of the order of the polynomial $R, S$ and $T$ divided by 10 ) in order to obtain a joint performance/complexity criterion. In Table 4 the controllers are compared with these criteria. It is observed that the controller designed by Method B has a good result compared with the other $H_{\infty}$ approaches.

\section{CONCLUSIONS}

Two different techniques, based on pole placement combined with sensitivity function shaping, have been presented. The first technique combines the main ideas in advanced robust control 


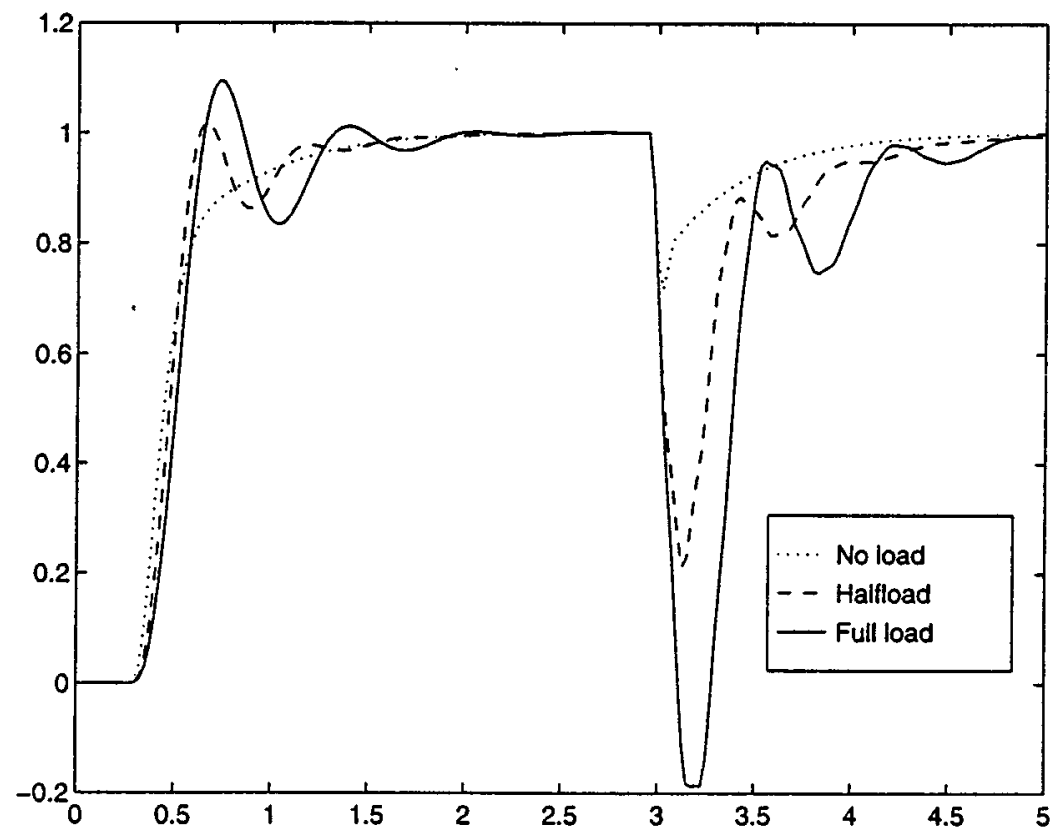

Figure 8. Step and disturbance response in simulation

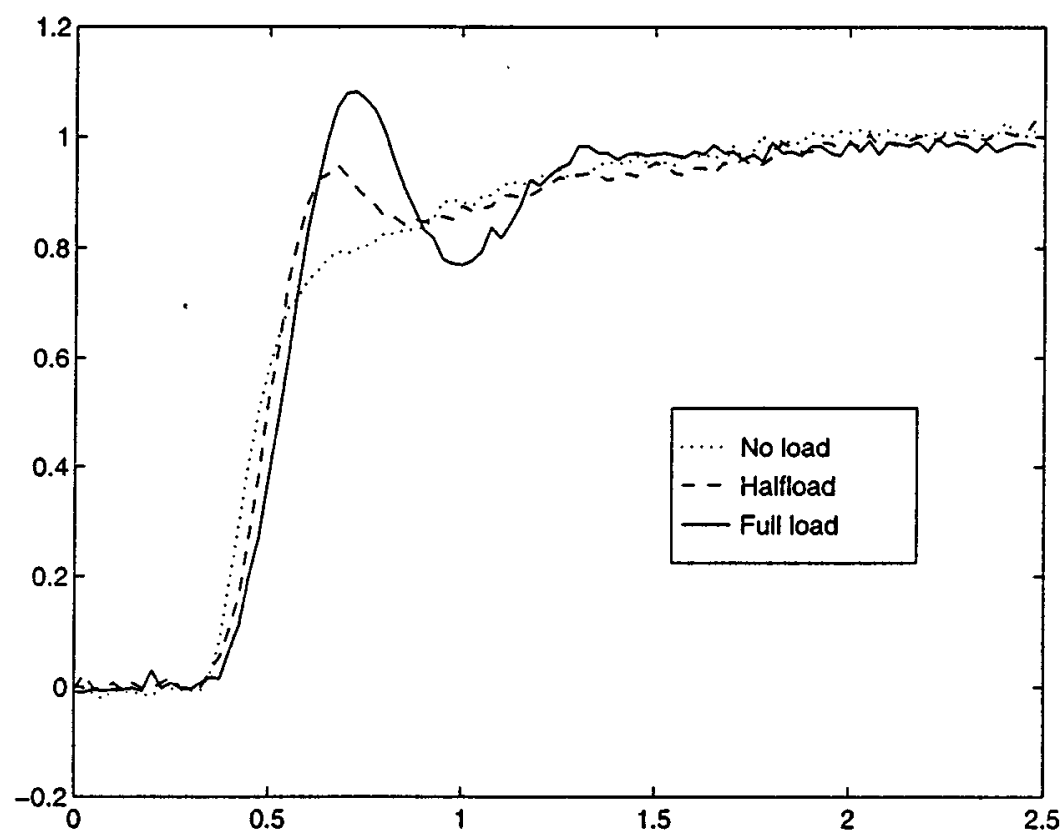

Figure 9. Step response in real time 
Table 1. Simulation results

\begin{tabular}{|c|c|c|c|c|c|c|c|}
\hline & $\begin{array}{l}\text { Rise time } \\
\text { (s) }\end{array}$ & $\begin{array}{l}\text { Over shoot } \\
(\%)\end{array}$ & $\begin{array}{l}\text { Rej. of dist. } \\
\text { (s) }\end{array}$ & $\begin{array}{l}\text { Delay } \\
\text { margin (s) }\end{array}$ & $\begin{array}{l}\text { Modulus } \\
\text { margin }(\mathrm{dB})\end{array}$ & $\begin{array}{l}\text { Atten. band } \\
\text { band }(\mathrm{Hz})\end{array}$ & $\begin{array}{l}\text { Maximum } \\
\text { of } S_{u p}(\mathrm{~dB})\end{array}$ \\
\hline No load & $1 \cdot 135$ & $0 \%$ & $1 \cdot 811$ & 50 & $-6 \cdot 45$ & $0 \cdot 118$ & $6 \cdot 7$ \\
\hline Half load & 0.643 & $1.6 \%$ & $1 \cdot 703$ & 345 & $-5 \cdot 29$ & $0 \cdot 119$ & $7 \cdot 2$ \\
\hline Full load & $0 \cdot 717$ & $9 \cdot 4 \%$ & $2 \cdot 105$ & 560 & -5.95 & $0 \cdot 116$ & 7.3 \\
\hline
\end{tabular}

Table 2. Experimental results

\begin{tabular}{lllll}
\hline & Rise time (s) & Overshoot $(\%)$ & Settling time (s) & Rej. of dist. (s) \\
\hline No load & $1 \cdot 671$ & $0 \%$ & $2 \cdot 7$ & $2 \cdot 55$ \\
Half load & $0 \cdot 714$ & $0 \%$ & $3 \cdot 1$ & $1 \cdot 55$ \\
Full load & $0 \cdot 742$ & $8 \cdot 1 \%$ & $2 \cdot 1$ & $1 \cdot 75$ \\
\hline
\end{tabular}

Table 3. Comparison with other approaches in simulation

\begin{tabular}{llr}
\hline Controller & Performance $(\%)$ criterion & Complexity (order of $R+S+T)$ \\
\hline Method A & $97 \cdot 12$ & 12 \\
Method B & $85 \cdot 09$ & 6 \\
Kwakernaak & $88 \cdot 80$ & 18 \\
Jones et al. & $94 \cdot 38$ & 35 \\
Walker & $72 \cdot 35$ & 15 \\
\hline
\end{tabular}

Table 4. Comparison with other approaches in real-time application

\begin{tabular}{lll}
\hline Controller & $\begin{array}{l}\text { Normalized sum of time } \\
\text { characteristics }\end{array}$ & $\begin{array}{l}\text { Joint performance/complexity } \\
\text { criterion }\end{array}$ \\
\hline Method A & $10 \cdot 86$ & $12 \cdot 06$ \\
Method B & $13 \cdot 64$ & $14 \cdot 24$ \\
Kwakernaak & $12 \cdot 71$ & $14 \cdot 51$ \\
Jones et al. & $11 \cdot 47$ & 14.97 \\
Walker & $22 \cdot 63$ & $24 \cdot 10$ \\
\hline
\end{tabular}

with the classical pole placement for SISO systems without using complex calculations. It adjusts iteratively the sensitivity functions in the frequency domain where it is necessary. Therefore, it seems that this technique is easy to understand for practising control engineers who are not familiar with the advanced robust control theories. This technique has already been applied to several real and industrial systems.

In the second technique, the idea of pole placement combined with sensitivity function shaping has been transferred to $H_{\infty}$ optimal control via a new interpretation of the weighting filter, as the inverse of the desired sensitivity function. In this technique, weighting filter selection, which seems 
to be the most important step in the $H_{\infty}$ design, is carried out automatically by an optimization program. Using this method a solution to a benchmark problem has been given, tested on a real system and compared with other $H_{\infty}$ type designs.

Research is in progress concerning the proof of the convexity of the optimization procedure for building the desired sensitivity function via a different parametrization.

\section{REFERENCES}

1. Landau, I.D., F. Rolland, C. Cyrot and A. Voda, 'Digital robust control. The combined pole placement/sensitivity shaping method', Presented at the Summer Control School of Grenoble 'Robustness Analysis and Design of Robust Controllers'. French version published in La Robustesse, A. Oustaloup (Ed), Hermes, Paris, 1993.

2. Landau, I.D., C. Cyrot and D. Rey, 'Robust control design using the combined pole placement/sensitivity function shaping method', European Control Conference, Gronigen, The Netherlands, 1993.

3. Landau, I.D., A. Karimi, A. Voda and D. Rey, 'Robust digital control of flexible transmission using the combined pole placement/sensitivity function shaping method', European Journal of Control, 1(2), (1995).

4. Fenot, C., F. Rolland, G. Vigneron and I.D. Landau, 'Open loop adaptive feedback control of deposited zinc in hot dip galvanizing, Control Engineering Practice, 5 (1993).

5. Zames, G. and B.A. Francis, 'Feedback, minimax sensitivity, and optimal robustness', IEEE Trans. Automat. Control, 28 (1983).

6. Francis, B.A. and G. Zames, 'On $H_{\infty}$ optimal sensitivity theory for SISO feedback systems', IEEE Trans. Automat. Control, 29 (1984).

7. Kwakernaak, H., 'A polynomial approach to minimax frequency domain optimization of multivariable systems', Int. J. Control, 44 (1986).

8. Postlethwaite, I., M.C. Tsai and G.D.W., 'Weighting function selection in $H_{\infty}$ design', 11-th IFAC World Congress, Tallian, 1990.

9. Doyle, C.J., B.A. Francis and A.R. Tannenbaum, Feedback Control Theory, McMillan, NY, 1992.

10. Kwakernaak, H., 'Robust control and $H_{\infty}$ optimization', Automatica, 29 (1993).

11. Landau, I.D., 'Robust digital control of systems with time delay (the Smith predictor revisited)', Int. J. Control, 62(2), (1995).

12. Landau, I.D., System Identification and Control Design, Prentice Hall, NJ, 1990.

13. Krause, J.M., 'Comments on Grimble's comments on Stein's comments on roll off of $H_{\infty}$ optimal controllers', IEEE Trans. Automat. Control, 37 (1992).

14. Sung, H.K. and S. Hara, 'Properties of sensitivity and complementary sensitivity functions in single-input singleoutput digital control systems', Int. J. Control, 48 (1988).

15. M'Saad, M., M. Duque and S. Hammad, 'Flexible mechanical structures adaptive control, in Adaptive Control Strategies for Industrial Use, C. Shah and G. Dumont (Ed), Springer Verlag, Berlin, 1989.

16. Landau, I.D., D. Rey, A. Karimi, A. Voda and A. Franco, 'A flexible transmission system as a benchmark for robust digital control', European Journal of Control, 1(2), (1995).

17. Kwakernaak, H., 'Symmetries in control system design', in Trends in Control A. Isidori (Ed), Springer Verlag, London, 1995.

18. Francis, B.A., 'A course in $H_{\infty}$ control theory', in Lecture Notes in Control and Information Sciences, number 88, Springer Verlag, Heidelberg, 1987.

19. Jones, N.W. and D.J.N. Limebeer, 'A digital $H_{\infty}$ controller for a flexible transmission system', European Journal of Control, 1(2), (1995).

20. Walker, D.J., 'Control of a flexible transmission — a discrete time $H_{\infty}$ approach', European Journal of Control, 1(2), (1995). 